\title{
DIE FEMME FATALE
}

Exemplarische Untersuchungen zu einem ästhetischen Weiblichkeitstypus im Fin de siècle

Inauguraldissertation

zur Erlangung des Grades eines Doktors der Philosophie im Fachbereich Neuere Philologien der Johann Wolfgang Goethe-Universität

zu Frankfurt am Main

vorgelegt von

Carola Hilmes

aus Eschwege 
1. Gutachter: Prof. Dr. Ralph-Rainer Wuthenow

2. Gutachter: Prof. Dr. Norbert Altenhofer

Tag der Promotion: 11. 2. 1988

Erschienen im Metzler Verlag 


\section{Carola Hilmes}

\section{DIE FEMME FATALE}

Ein Weiblichkeitstypus in der nachromantischen Literatur

J. B. Metzlersche Verlagsbuchhandlung Stuttgart 
CIP-Titelaufnahme der Deutschen Bibliothek

Hilmes, Carola:

Die Femme fatale : ein Weiblichkeitstypus in der nachromantischen Literatur / Carola Hilmes.

- Stuttgart : Metzler, 1990

ISBN 978-3-476-00691-2

D. 30

ISBN 978-3-476-00691-2

ISBN 978-3-476-03307-9 (eBook)

DOI 10.1007/978-3-476-03307-9

Dieses Werk einschließlich aller seiner Teile ist urheberrechtlich geschützt. Jede Verwertung außerhalb der engen Grenzen des Urheberrechtsgesetzes ist ohne Zustimmung des Verlages unzulässig und strafbar. Das gilt insbesondere für Vervielfältigungen, Übersetzungen, Mikroverfilmungen und die Einspeicherung und Verarbeitung in elektronischen Systemen.

(C) 1990 Springer-Verlag GmbH Deutschland

Ursprünglich erschienen bei J. B. Metzlersche Verlagsbuchhandlung und Carl Ernst Poeschel Verlag GmbH in Stuttgart 1990 
Meinen Eltern 


\section{Inhalt}

\section{VORWORT}

Seite X

\section{METHODISCHE ANNÄHERUNG AN DAS "RÄTSEL-WEIB»

\author{
Seite 1
}

\section{DÄMONISIERUNG DES WEIBLICHEN IM FIN DE SIECLE \\ Konstitutionsbedingungen und Bedeutungshorizont der Femme fatale}

Seite 14

Mortifizierte Weiblichkeit als Verbindung von Romantik und Décadence: Eurydikemythos bei Novalis 17 . Schlegels »Lichtbringerin « 21 . Femme fatale und Femme fragile: Surrogate eines sublimierten Eros 28 - Dialektik idealisierter Weiblichkeit bei E. T. A. Hoffmann: Julie und Giulietta 33

$$
\text { Exkurs: Foucault - Freud - Weininger }
$$

Seite 39

Michel Foucault, Diskursivierung der Sexualität 39 - Sigmund Freud, Weiblichkeit als »Defekt« und »Infekt« 42 . Otto Weininger, Der geschlechtliche Charakter der Frau 45

\section{Das Bild der »sinnlichen Frau« im Fin de siècle}

\section{Seite 51}

Veränderung der Familienstruktur und das Beharrungsvermögen der Imaginationen 51 . Die sinnliche Frau als Fluchtprojektion einer subkulturellen Gegenöffentlichkeit 55 . Wilhelm Arent und Felix Dörmann, "Satanella« und »Madonna Lucia«61 - Die Femme fatale als Spiegel einer Krisis des (männlichen) Selbstbewußtseins 65 . Geschichten der tödlichen Gefahren eines instrumentellen Eros 67 . Ein trügerisches Modell weiblicher Emanzipation und die Subversion der Bilder 71 


\section{JUDITH UND DELILA}

Rekonstruktion der Sinnlichkeit als weiblicher Macht und der chimärenhafte Charakter der Femme fatale Seite 74

Johanna - eine Frau betritt den imaginären Raum der Geschichte Seite 76

Nicht alle Jungfrauen in Waffen sind gleich

Seite 80

Judith - symbolisches Geschichtsdrama und psychologische Geschlechtertragödie Seite 83

Grotesker Epilog - Die jüdische Witwe Seite 93

Delila - prototypische Hohlform der Femme fatale Seite 94

\section{GESCHICHTEN DER SALOME: BLUTIGE BILDER DER LIEBE}

Ästhetizistische Varianten idealisierter Weiblichkeit und der patriarchalische Blick

Seite 102

Oscar Wilde: »Salome«, eine Geschichte tödlicher Blicke Seite 111

Heinrich Heine: »Herodias«/»Salome« im letzten freien Waldlied der Romantik Seite 122

Gustave Flaubert: »Herodias«, kritisch-analytischer Blick ernüchterter Romantik

Seite 128

Oskar Panizza : »Salomes Tochter«, eine vergessene Variante Seite 139

Stéphane Mallarmé: »Hérodiade«, Femme fatale étonnante et étrange Seite 144 


\section{LULU - RAFFINIERTER VAMP UND MODERNE HETÄRE Zu Wedekinds Konstruktion "freier Sinnlichkeit"} Seite 155

\section{ALLEGORISCHE METAMORPHOSEN DES WEIBLICHEN Zu Heinrich Manns Roman "Die Göttinnen"} Seite 177

Die Herzogin als Femme fatale 180 - Botticellis »Primavera« als erkenntnistheoretisches Medium 182 - Diana: Politik als Operette und Geschäft - Dilettantismus als Haltung 186 · Minerva: Das Verhältnis von Kunst und Liebe · Inszenierung als künstlerische Lebenspraxis 189 . Masochismus als spiritueller Kult der Sinne 194 - Venus Anadyomene und Madonna Verderberin: Vision idealer Liebe und Bild dämonischer Weiblichkeit 196 - Venus: Synthese von Liebes- und Todesgöttin $200 \cdot »$ Heidnische Sinnlichkeit« • Unterschiede zwischen Wedekind und Heinrich Mann 202 · Heinrich Manns "positives« Frauenbild $205 \cdot$ Ästhetisch-praktischer Pessimismus und der Traum vom Ich 210 · Yollas Lächeln - imaginäre Versöhnung 213 · Moderne Form : Zitatmontage, Allegorie und leere Identität 217

\section{MOTIVE UND MERKMALE Seite 223}

Rache-, Opfer- und Tötungsmotive

Seite 223

Aufspaltung, Verknüpfung und Verschiebung von Motiven. Der ungreifbare, chimären- und rätselhafte Charakter der Femme fatale Seite 231

Unheilvolle Verbindung von Narzißmus und Voyeurismus Seite 237

Bildlichkeit - Interdependenz von Literatur und Malerei Seite 240

Künstlichkeit und tödliche Liebe Seite 244

Dissonanzen - Probleme der Rezeption

Seite 247 


\section{LITERATURVERZEICHNIS}

Seite 249

VERZEICHNIS DER ABBILDUNGEN

Seite 263 


\section{Vorwort}

"Wenn du mich liebst, bist du erledigt«, sagt Godards Carmen und gibt damit eine kurze, aber präzise Charakteristik der Femme fatale. Dieses suggestiv stark aufgeladene Bild des Weiblichen, das auch den Namen »dämonische Verführerin « trägt oder »la belle dame sans merci« heißt, hat seit einiger Zeit wieder Konjunktur. Immer häufiger blickt uns eine herzlose Schöne aus den Werbeseiten der Illustrierten an. Das ehemals sibyllinische Lächeln ist einem kühlen, ja verachtenden Blick gewichen. Das Unnahbare und Undurchschaubare machen diese Frauen jedoch nur um so anziehender. Geworben wird mit dem Bild der ewigen Verführerin, die Exklusivität verspricht und Überraschungen, böse Überraschungen und leidenschaftliche Erfahrungen, die nicht ganz ungefährlich sind.

Bereits der durchschlagende Erfolg von Sauras Carmenfilm 1983 war ein Indiz für die Sehnsucht nach "neuer « Intensität, nach heftigen Gefühlen, die sich nicht regulieren lassen durch lange »Beziehungsdiskussionen«. Carmens Liebe duldet keinen Aufschub, verweigert sich dem Kalkül, jeder Ökonomie der Gefühle. Wenn Carmen liebt, liebt sie bedingungslos, bedenkenlos, verschwenderisch. Das macht sie so faszinierend wie bedrohlich.

Auch »Die flambierte Frau«, das wenig realistische Porträt einer Edelprostituierten, die als Eva/Carmen eine Doppelexistenz führt, unterstreicht den Trend zur Femme fatale im Kino. Dieser Film machte die Domina in weiten Kreisen diskussionsfähig. Die Schattenseiten der Sexualität wurden ans Licht gezerrt. Erneut begann das Rätselraten um die geheimnisvolle, nicht in Besitz zu nehmende Frau, die durch ihre "Aura fesselt und durch ihre Abgründigkeit erschreckt. Ob Carmen ihre Würde und Freiheit verteidigt und die Eigenständigkeit der flambierten Frau eine mögliche Form der Emanzipation darstellt, bleibt allerdings zweifelhaft.

Robert van Ackerens neuester Film trägt wieder einen vielversprechenden Titel, »Die Venusfalle«. Erzählt wird nicht mehr eine Geschichte von der Last der Lust, sondern von den Omnipotenzphantasien eines Mannes - eines Zwangsromantikers, der, in ständiger Angst, die Frau seines Lebens zu verpassen, den Rekord im Seitenspringen hält, bis endlich die Falle der Liebe zuschnappt und er sich in eine verhängnisvolle Affaire verwickelt. Eine ganz alltägliche Geschichte, ein postmoderner Dreigroschenroman, eine Zitatmontage aus Banalitäten.

$\mathrm{Daß}$ die um eine sog. Femme fatale sich rankende Geschichte weder originell noch konsistent sein muß, wird auch an dem Film »Fatal Attraction « von Adrian Lyne deutlich. Hier ist lediglich der Titel programmatisch. Darüber hinaus macht der Film wie kaum ein anderer klar, daß eine Femme fatale - so die blonde Protagonistin denn eine sein soll - mit entfesselter Erotik nichts zu tun hat, die Konzeption dieses Klischees vielmehr darauf hinausläuft, die Ideologie von der glücklichen Familie zu stärken. In eindeutig moralisierender Absicht wird ge- 
warnt vor dem Seitensprung und seinen fatalen Folgen. Daß die Geliebte eines Wochenendes völlig überzogen reagiert, ihre Versuche, den verheirateten Mann für sich zu gewinnen, weitgehend unmotiviert bleiben, scheint die meisten Zuschauer nicht weiter gestört zu haben. Die Suggestionskraft des Klischees reicht offenbar aus, eine haarsträubende Geschichte zusammenzuhalten und sie zu einer akzeptierten Geschichte zu machen.

Ebenfalls vom Titel her programmatisch ist der Film »Ein mörderischer Engel« mit Charlotte Rampling, die seit ihrer spektakulären Rolle in Liliana Cavanis »Nachtportier« 1973 als die Femme fatale des Kinos der letzten Jahre gilt. Sie spielt Frauen in extremen Situationen, Frauen, die undurchschaubar und nicht verfügbar sind, Frauen, die bei Männern bizarre Lüste wecken, wie Mareike Boom schreibt. Sie lockt mit einem vielsagenden, von schweren Lidern überschatteten Blick und ihrem schönen Körper. Ein geheimnisvolles Flair umgibt sie. Ihr Image wird aufgeladen mit den dunklen Frauenfiguren aus der Mythologie. In einem Porträt in "Vanity Fair « heißt es: »Charlotte Rampling is everyone's favorite sphinx, nemesis, siren, and general bad girl. «

Die Geschichte mit dem »mörderischen Engel« geht gut aus - für den Mann zumindest. Inspektor Staniland bringt Barbara (Charlotte Rampling) ins Gefängnis. Nicht unbedingt führt die Begegnung mit einer Femme fatale zum Ruin. Oft kommen die Männer mit dem Schrecken und einigen Blessuren davon. Die dämonische Verführerin aber, der schwarze Engel, wird seiner »gerechten « Strafe zugeführt. Für die Femme fatale endet die Geschichte meist tödlich. Sie wird nicht nur den Männern zum Verhängnis. Ein selten bemerktes Charakteristikum dieses Typus. Sollte es so selbstverständlich sein, daß es noch nicht einmal Erwähnung findet?

Aufschlußreich ist Charlotte Ramplings letzter Film »Paris by Night«, der in diesem Frühjahr auch in den deutschen Kinos anlaufen wird. In diesem Politthriller von David Hare spielt die Rampling eine unantastbare Karrierefrau mit Geheimnis. Clara Page ist Abgeordnete des Europaparlaments, eine Frau, die die konservative Ideologie ihrer Partei auch auf ihr Privatleben überträgt: Konsequenz, Härte, Erfolg. Bedürfnis nach Zärtlichkeit hat sie nur in schwachen Stunden, und selbst dann weiß man nicht, wieviel Berechnung im Spiel ist. Sie ist eine hartgesottene Lady, damenhaft gekleidet, aber zielstrebig und stets geschäftsmäBig - der europäische Frauentyp der Nachhollywood-Ära? Die moderne Geschäftsfrau in der Tradition der Femme fatale?

Anläßlich der Premiere von »Paris by Night« im letzten Sommer schrieb der Guardian: »Ms Rampling, the latest version of the British femme fatale«. Erstaunlich ist die Wandlungs- und Anpassungsfähigkeit dieses quasi mythischen Frauenbildes. Die Femme fatale, Grenzgängerin und Grenzüberschreiterin, erscheint heute wieder als die »wahre« Frau: Unangepaßt und eigenwillig, streng durchgestylt und cool kalkuliert sie eiskalt den eigenen Vorteil und erobert männliches Terrain. Die moderne Hexe präsentiert sich als obsessive Karrierefrau. Getrieben von einem Willen zur Macht ist sie bereit, über Leichen zu gehen. Ihre Attraktivität ist dabei lediglich Fassade, der Wunsch nach Leidenschaft und Erotik eine männliche Projektion. Das Gefährliche schiebt sich in den Vordergrund, die 
Angst vor der Konkurrentin und den Unwägbarkeiten ihrer Macht. In ihrer aktuellen Erscheinungsform ist die Femme fatale nicht mehr so sehr dämonische Verführerin als diabolisches Weib schlechthin. Ihre erotische Macht ist zwar ungebrochen, bildet aber jetzt nur noch ein Element im Kampf der Geschlechter, das, von den Männern vollends unkontrollierbar, für eigene Zwecke eingesetzt wird. War die Femme fatale immer schon ein Gegenentwurf zum klassischen Bild des Weiblichen, so treten mit der Dysfunktionalität seine subversiven Kräfte jetzt deutlich hervor.

Eine kritische Analyse gibt Einblick in die Problematik und in die Sprengkraft dieses Weiblichkeitsbildes. Seine Aktualität, wie sie in der Werbung und im Kino zu beobachten ist, erscheint vielleicht in einem weniger bizarren Licht, wenn man nach den kulturhistorischen Entstehungsbedingungen dieser Imago fragt. $\mathrm{Da} ß$ die Femme fatale keinen Realtypus des Weiblichen bezeichnet, sondern ins Reich der kollektiven Phantasien gehört, wird dabei vorausgesetzt - auch wenn eine entsprechende Selbststilisierung von Frauen dem zu widersprechen scheint. Die Anziehungskraft dieser Imago für Frauen ist einerseits verständlich - wer wollte sich nicht mit der geheimnisvollen Schönen und der ihr zugeschriebenen Macht identifizieren? Andererseits erweisen sich aber entsprechende Selbststilisierungen als verhängnisvoll. Ob dabei die diesem Klischee innewohnende selbstzerstörerische Komponente aus Unkenntnis ignoriert oder als subversive Strategie bewußt in Kauf genommen wird, muß offenbleiben. Solange die Herrschaft der schicksalhaften Bilder des Weiblichen nicht wirklich gebrochen ist, vermag nur in ihrer Schönheit ein utopischer Rest sich zu tradieren. Vielleicht hieße Emanzipation nicht zuletzt eine Befreiung von diesen verhängnisvollen, defizitären Vorstellungen.

Ist auch die Zeit der großen ästhetischen Lebensentwürfe vorbei, so funktioniert die quasi mythische Überhöhung der Realität bis heute. Nun orientiert man sich am Celluloid Image und an der Hochglanzillustrierten. Die Metamorphosen ins Alltägliche erweisen das suggestive Bild der Femme fatale als das, was es bereits um 1900 war: einen Trivialmythos von irritierender Doppelbödigkeit. Was heute als später Abklatsch erscheint, der in seiner ideologischen Stoßrichtung leicht zu durchschauen, in seiner Disparatheit aber schwer zu erfassen ist, läßt sich, nicht zuletzt aufgrund zeitlicher Distanz, in der nachromantischen Literatur besser begreifen.

Dort heißen sie Judith und Delila, Lulu, Salome und Satanella, die rätselhaften Schönen, um die sich eine abgründige Geschichte rankt. Bis zur Unkenntlichkeit können die erzählten Geschichten die mythologische oder biblische Vorlage verändern. Zuweilen wird lediglich noch der Name zitiert oder ein markantes Motiv aufgegriffen, wie z. B. das des abgeschlagenen Kopfes. Ob die Übeltäterin Judith ist oder Salome, wird so nicht mehr klar, ist aber offensichtlich auch nicht entscheidend. Charakteristisch für eine Femme fatale ist nur die Übermächtigungssituation. Selbst auf eine kurze triumphale Siegerpose des vermeintlich diabolischen Weibes kann verzichtet werden, wenn nur die Begegnung zwischen Mann und Frau ein verhängnisvolles Ende nimmt und die traditionellen Herrschaftsverhältnisse zwischenzeitlich verkehrt worden sind. Dank ihres chimären- 
haften Charakters sind alle einzelnen Merkmale austauschbar. Die extreme Wandlungsfähigkeit der Femme fatale, die negativ als Gesichtslosigkeit gefaßt werden kann, erklärt positiv gesehen ihre Dauerhaftigkeit.

$\mathrm{Da}$ es bei der Disparatheit ihrer Erscheinungsformen überhaupt möglich ist, die verschiedenen Figuren als Femme fatale zu identifizieren, liegt daran, daß die dämonische Verführerin in gewisser Weise ein Urbild verkörpert. Sie repräsentiert eine ambivalent besetzte Sexualität, mit der das Weibliche gleichgesetzt wurde und oft immer noch wird. Die Hoffnung auf Glück, das dieser Figur stets anhaftende utopische Moment, erscheint jedoch aufs engste verbunden mit der Angst vor der (männlichen) Selbstaufgabe, einem Verzicht auf bürgerliche Saturiertheit und eine Identität, deren Zwänge auch Halt und Stütze sein können. Die Femme fatale markiert das Andere der Vernunft und bleibt doch dem patriarchalischen Diskurs verhaftet. Dieser so großartig erscheinenden Figur liegt eine kleinmütige Kalkulation zugrunde, voller Ressentiment und Vorurteil. Es ist bloß eine Koketterie mit dem Begehren - ein schauerlich schöner Wunschtraum des Spießers. Aus der sicheren Position des Zuschauers ruht sein Blick wohlgefällig auf dem Satansweib.

Als unter dem männlichen Blick sich konstituierendes Bild kann die Femme fatale nicht verschleißen. Eine in kritischer Absicht vorgenommene Dekomposition, die die misogynen Vorurteile und defizitären Bestimmungen des Weiblichen aufzeigt, erweist sich als Sisyphosarbeit. Die Femme fatale ist eine Fata Morgana, die ihre Suggestionskraft auch dann nicht verliert, wenn man um die Bedingungen der Luftspiegelung weiß. Als Projektionsfigur ist sie nicht zu eliminieren. Was wäre auch zu greifen?

Carmen und Lulu, Salome und Judith - die Namen sind so einprägsam wie die mit den Figuren verbundene Geschichte dunkel. Die erotische Phantasie vermag sich an fragmentarischen Szenen am besten zu entzünden. Sie heftet sich ans Bild der dämonischen Verführerin. Wie kaum eine andere Figur ist die Femme fatale auf Visualisierung angewiesen. Die Interdependenz von Malerei und Literatur ist für sie von besonderer Bedeutung. Während auf einem Bild eine einzelne Szene dargestellt wird und der Betrachter sich seine Geschichte der Femme fatale imaginieren kann, steht eine literarische Bearbeitung vor dem Problem, einen möglichst konsistenten Handlungszusammenhang herstellen zu müssen. Die Geschichten der Femme fatale sind verräterisch, weil sie sich nicht beschränken können auf die für das Klischee typische Situation der Übermächtigung eines Mannes durch weibliche Sinnlichkeit. Die Brüchigkeit dieser Imago läßt sich noch am mangelnden Zusammenhang und der ungenügenden Motivation ablesen. Hierin sind die »klassischen « Varianten aus der nachromantischen Literatur den Filmen von heute durchaus verwandt. Das Werbebild rückt neben das Ölgemälde.

Behelfen wir uns vorab mit einem kurzen »Steckbrief« dieses Phantoms: Die Femme fatale lockt, verspricht und entzieht sich. Zurück bleibt ein toter Mann. Im Spannungsfeld von Eros und Macht gedeihen Wollust und Grausamkeit, entstehen blutige Bilder der Liebe. Die Femme fatale fasziniert durch ihre Schönheit und das in ihr liegende Versprechen auf Glück, einen Wunsch nach leidenschaftli- 
cher Liebe. Gleichzeitig wird sie jedoch auch als bedrohlich empfunden. Die Gefahr geht aus von der in ihr verkörperten Sexualität und der Einbindung in eine Geschichte voller Intrigen, in der ihr meist die wenig rühmliche Rolle einer Rächerin zugeschrieben wird. Die Femme fatale repräsentiert die permanente Verführung, die ebensosehr gewünscht wie gefürchtet wird. Diese Doppelbödigkeit macht sie so geheimnisvoll wie unheimlich. Die der Femme fatale zugeschriebene Dämonie hat ihren Grund nicht in einer bestimmten diabolischen Eigenschaft, einem potentiell allen Frauen gemeinsamen Wesensmerkmal, sondern in dieser zwiespältigen Disposition als Wunsch- und Angstbild.

Die Femme fatale eröffnet gerade keinen Blick auf die Frauen. Die heimlichen Helden der Geschichten sind ihre männlichen Gegenspieler. So kann das Weib nur deshalb übermächtig erscheinen, weil ihr Gegenspieler in einer schwachen und verunsicherten Position ist. Dem Torero hätte Carmen nicht zum Verhängnis werden können. Die Femme fatale spiegelt das Prekäre der männlichen Situation. In Zeiten entsprechender Verunsicherung und Orientierungslosigkeit hat dieses Bild Konjunktur. Das trifft zweifellos für das allgemeine Krisenbewußtsein um 1900 zu. Daß es gleichermaßen für unsere Zeit gilt, die sog. Postmoderne, ist wahrscheinlich.

Die Figur der Femme fatale enthält eine implizite Kritik. Kritik nicht nur an der prekären Situation des Mannes, seiner Ichschwäche und verdrängten Triebverfallenheit, sondern auch Kritik an der traditionell untergeordneten Situation der Frau, ihrer Beschränkung auf eine passive und asexuelle Rolle in der bürgerlichen Gesellschaft. Daß die patriarchalischen Rollenzuweisungen mit der Femme fatale durchbrochen werden, macht diese Figur auch für Frauen attraktiv. Die Femme fatale kann interpretiert werden als ein Bild der Rebellion gegen die klassischen Imaginationen des Weiblichen, die positiv besetzten Bilder von der Jungfrau und Mutter sowie das negativ belastete der Hure. Es markiert eine Befreiung aus einem marginalen, bloß gefühlsseligen Dasein, dem kein öffentlich relevanter Handlungsspielraum zukommt. Mit der Femme fatale wird den Nachtseiten der Sexualität und des Weiblichen Raum gegeben. Insofern kommt ihr ein gewisses subversives Potential zu.

Trotzdem taugt die Femme fatale nicht als weibliche Freiheitsphantasie. Die Begrenzung weiblicher Macht auf den Bereich der Erotik ist verhängnisvoll. Sieht man sich die Geschichten der Femme fatale in der nachromantischen Literatur an, muß man erkennen, daß der ihr zugestandene Handlungsspielraum nicht nur begrenzt ist, sondern auch ein geliehener. Nur unter der Herrschaft des männlichen Blicks vermag die Femme fatale ihre Macht zu entfalten. Der errungene Triumph ist ein wahrer Pyrrhussieg. Daß die dämonische Verführerin für die ihr unterschobene Wollust und Grausamkeit am Ende der Geschichte stets abgeurteilt wird, stellt dabei eine doppelte Denunziation der Frauen dar. Die in diese Imagination eingegangenen misogynen Voraussetzungen fordern am Schluß wieder ihre angestammten Rechte ein.

Angesiedelt im Spannungsverhältnis von erotischer Faszination und bedrohlicher Macht, spiegeln sich in dem aus unterschiedlichen Komponenten zusammengesetzten Bild der Femme fatale sowohl Anpassung an das traditionelle 
Verhältnis der Geschlechter als auch Dissens zu ihm. Heute, wo die Verfügung über das mythische Arsenal grenzenlos geworden ist, sind die Ambivalenzen dieser Imago, das Doppelbödige und Widersprüchliche, weitgehend ausgelöscht. Im Zeitalter des Bildertriumphes und der universellen Zitierbarkeit wird auch die Femme fatale zum Verkaufsschlager. Die Veralltäglichung hat den Mythos soweit entleert, daß er nun schadlos konsumierbar geworden ist. Die subversiven Elemente, die mit dieser Imago um 1900 noch verbunden waren, sind eliminiert, das anarchistische Moment der Sexualität stillgestellt - ein Entkopplungsmanöver im Dienste eines erzwungenen Pragmatismus.

Die aseptischen Videos der Popsängerin Madonna, in denen die ganze Palette der Femme fatale vorgeführt wird, sind das beste Beispiel für die völlige Banalisierung dieses Bildes. Die extreme Stilisierung zur dämonischen Verführerin in all ihren Varianten ist hier stets verbunden mit einer Abwehr der bedrohlichen sexuellen Reize. Die Inszenierung ist perfekt, aber harmlos. Über eine solche Versachlichung als Folge der Entmythifizierung muß man nicht traurig sein. Die Domestizierung und Vermarktung der Femme fatale, dieser ehemals zwiespältigen und sperrigen Männerphantasie, in der heute die zerstörerischen Seiten völlig ausgeblendet sind, stellt auch eine Form positiver Demokratisierung dar. Was sonst nur den exklusiven Bohèmezirkeln vorbehalten war, wird zum Massenartikel.

Heute dominiert der Unterhaltungswert dieser nur noch dämonisch erscheinenden Figur, deren ästhetische Reize von den Medien weidlich ausgeschlachtet werden. Eine solche Vereinnahmung kann funktionieren, weil mythische Bilder ungeheuer belastbar sind. Heute dienen sie vornehmlich als Kostüm. Auch Maria von nebenan kann sich dieses selbstgewählte Kleid überstülpen. Heute Abend tragen wir Femme fatale. Selbst Frauen, die sich sonst gegen eine sexistische Vereinnahmung wehren, schlüpfen gern in diese Maske, die zu einer beliebigen Tauschmünze geworden ist. Diese größere Offenheit ist nicht zu beklagen, ermöglicht sie doch ein freies Spiel ohne verhängnisvolle Folgen.

Sogar eine Umkehrung der Situation wird nun denkbar: vom Märchenprinzen zum homme fatal, der Wunschphantasie vom starken und leidenschaftlichen Mann, dessen Unberechenbarkeit man/ frau sich gerne ausliefert - in der Phantasie. In dem Film »Neuneinhalb Wochen «, diesem letzten Renner unter den Softpornos, der mit glatten und keimfrei deodorierten Bildern eine sadomasochistische Liebesgeschichte erzählt, reagiert die von Kim Basinger gespielte Heldin auf einen homme fatal, der sie dazu bringt, eine gefährliche Weiblichkeit zu inszenieren. Der Maskenball ist total geworden. In der einschlägigen Presse wurde dieser Film als Beispiel einer leidenschaftlichen Beziehung gefeiert - und gleichzeitig davor gewarnt. Das Kino als Traumfabrik einer verwalteten Welt, die sich Pannen nicht leisten kann.

Das »wahre « Erbe der Femme fatale tritt die moderne Karrierefrau an - nicht die reale Geschäftsfrau natürlich, sondern das Bild von ihr, wie wir es alle aus dem »Denver Clan « kennen. Was Alexis zur aktuellsten Form der Femme fatale macht, ist die von ihr ausstrahlende Kraft, die sich dem männlichen Willen nicht fügt. Sie sucht die Konfrontation auf dem ureigensten männlichen Terrain, der Geschäfts- 
welt. Daß sie im Kampf um Geld und Macht die Waffen einer Frau einsetzt, macht sie so unberechenbar und überaus gefährlich. Die von ihr bei den Männern evozierten Ängste liegen darin, nicht mehr den eigenen Ansprüchen zu genügen. Das betrifft das selbstgebaute Lebensmodell und ist nicht mehr ins Reich der Mythen zu verweisen. Machten die »klassische « Femme fatale und ihr Zitat im Film sowohl den Wunsch nach leidenschaftlichem Liebesverlangen als auch das Ruinöse seiner Verwirklichung deutlich, so steht nun die Unmöglichkeit partnerschaftlichen Umgangs zur Debatte. Das Bild der modernen Karrierefrau suggeriert, daß der Kampf der Geschlechter erst jetzt wirklich begonnen hat.

Wie die »klassische « Femme fatale ist ihre letzte Verwandlung kein durchsetzungsfähiges und verallgemeinerbares Modell, sondern die Ausnahme - ein angstbesetztes Phantasieprodukt von zweifelhaftem Reiz. Barg die »Aura der Femme fatale noch das Versprechen auf Glück, so beschränkt sich die Ausstrahlung der Karrierefrau auf die Erotik der Macht. Nicht der Einsatz aller männlichen Weiblichkeitsphantasien macht sie so bedrohlich, sondern die Macht, über die sie neben ihrer erotischen Attraktivität noch verfügt. Die subversiven Elemente sind kassiert. Es herrscht die nackte Angst. Die Geschichte der Femme fatale ist noch nicht zu Ende. 